\title{
Attitudes to publicly funded obesity treatment and prevention
}

Lund, Thomas Bøker; Sandøe, Peter; Lassen, Jesper

Published in:

Obesity

DOI:

10.1038/oby.2011.84

Publication date:

2011

Document version

Peer reviewed version

Citation for published version (APA):

Lund, T. B., Sandøe, P., \& Lassen, J. (2011). Attitudes to publicly funded obesity treatment and prevention. Obesity, 19(8), 1580-1585. https://doi.org/10.1038/oby.2011.84 


\title{
Attitudes to publicly funded obesity treatment and prevention ${ }^{1}$
}

\author{
Thomas B Lund ${ }^{\S}$, Peter Sandøe*, Jesper Lassen ${ }^{\S}$
}

$\S$ Department of Human Nutrition, Faculty of Life Sciences, University of Copenhagen, Frederiksberg, Denmark

* Institute of Food and Resource Economics, Faculty of Life Sciences, University of Copenhagen, Frederiksberg, Denmark

Correspondence: Thomas B. Lund, Department of Human Nutrition, Faculty of Life Sciences, University of Copenhagen, Rolighedsvej 30, DK-1958 Frederiksberg C, Denmark. Telephone 0045353-33628, E-mail: (tblu@life.ku.dk)

\footnotetext{
${ }^{1}$ The reference of the printed version is:

Lund, Thomas B, Peter Sandøe \& Jesper Lassen (2011): Attitudes to Publicly Funded Obesity Treatment and Prevention. Obesity 19 (8): 1580-1585.

The definitive version is available at http://www.nature.com/oby/journal/v19/n8/full/oby201184a.html
} 


\begin{abstract}
The aim of this study was to investigate the Danish public's support for publicly funded obesity treatment and prevention. It was also examined whether levels of support could be explained by dislike of obese people and / or the belief that those who are obese are personally responsible for their condition. A representative survey of members of the Danish public $(\mathrm{N}=1,141)$ was conducted using a web-based questionnaire. The survey was designed to assess attitudes to public funding for obesityrelated health care, and to investigate the impact, on those attitudes, of dislike of obese people, the perceived controllability of obesity, self-reported BMI, and additional attitudinal and sociodemographic characteristics. Public funding of some obesity treatments, such as weight-loss surgery, attracted only limited public support. A majority of the Danish public did support 'softer' treatment interventions and preventive initiatives. Attitudes to the treatment of obesity were clearly best predicted by the belief that individuals are personally responsible for their own obesity. Dislike of obese persons had no direct effect on the preference for collective treatment initiatives and only a small effect on support for publicly funded obesity prevention. The high level of disapproval for publicly funded obesity treatment should be cause for concern for decision makers aiming to ensure equal access to health care. Since it is the belief that obese people are personally responsible which explains this disapproval, strategies for challenging public opinion on this issue are discussed.
\end{abstract}

Keywords: Prevention, Obesity Treatment, Public Policy, Prejudice, Weight-related Discrimination

\title{
Introduction
}

Obesity prevention and treatment is becoming an integrated part of health care in many countries. This development may come under public scrutiny in countries with collectively funded health care systems. Whether funding comes from general taxation, job insurance, private insurance or a combination of these, such systems share the feature that health care is not simply a private, financial matter between the individual patient and the service suppliers. Consequently, existing health care budgets and 
priorities may become a subject of public discussion (1). In this respect, it is relevant to examine public support for health care provision for obesity-related diseases within such contexts.

Previous studies of public attitudes have looked primarily at the public funding of obesity prevention. These studies have focused on support for information-based prevention (2-6), on regulation $(3,6,7)$, and on ways of inhibiting the development of obesity in childhood $(2-4,6,8,9)$. To our knowledge, no published studies to date have examined public support for obesity treatment. However, in view of the growing number of treatments available for severe obesity - including weight-loss surgery and medication, as well as dietary and behavioural therapies (10) - it is topical to examine the issue.

Equally, in this context a careful study of the causes of public approval and disapproval for treatment provision ought to provide a better understanding of the factors affecting levels of support.

In this respect, it is generally assumed that prejudicial attitudes to the obese may lead to discriminatory views and behaviours $(11,12)$. Most measures of prejudicial attitudes to obesity include a dislike dimension, although the content of this dimension varies considerably in empirical investigations (1218). Another dimension frequently included in measurements of prejudice centres on the belief that obesity is self-inflicted - something the individual is personally responsible for and is in a position to do something about $(12-15,17,18)$. We shall refer to these two dimensions as 'Dislike' and 'Perceived Control', respectively, capitalizing the terms to indicate that they are semi-technical.

These two dimensions of obesity prejudice have been shown to be strongly correlated $(12-15,17,18)$. Furthermore, in the so-called 'attribution-value model' they are depicted as interconnected factors in individual and group-based reasoning processes: the model links Perceived Control with resultant Dislike. The mechanism in play here opens with the initial identification of a negative attribution, in this case obesity. In combination with the judgment that obese people are personally responsible for their condition, this attribution leads to Dislike (19).

It is at present a relatively unresolved question to what extent Dislike and Perceived Control determine public attitudes to obesity-related health care. The few studies that exist - which, as mentioned above, 
examine public support only for prevention measures - report only a modest impact of Perceived Control $(5,6)$. Furthermore, in these studies measures of Perceived Control alone are included, making it impossible to infer anything about the combined effects of the prejudicial factors of Dislike and such control.

Against this background, the present study: (1) examined support for public funding of obesity treatment and prevention among the Danish public; and (2) investigated the predictors of support in order to identify its sources. Particular emphasis was placed on the role of the dimensions of Dislike and Perceived Control.

\section{Methods and procedures}

\section{Participants}

The data were collected by a commercial survey company which holds an internet database with entries for approximately 120,000 Danish citizens. From this database a stratified sample $(\mathrm{N}=3,696)$ was drawn to obtain a representative sample of Danish citizens aged 20-70. The sampled participants were invited to answer an internet-based questionnaire in February and March 2010. All participants who completed the questionnaire took part in a lottery in which three vouchers, each worth 1,000 Danish KR ( $\approx 135$ Euro), were drawn. In accordance with Danish legislation (20), an IRB approval was not applied for in this study, since all respondents were completely anonymous - even to the researchers and no experiments on humans or human biological material was carried out. 1,141 participants completed the questionnaire, resulting in a response rate of $30.9 \%$. The socio-demographic characteristics of the sample are presented in table 1. Compared to national census data (from Statistics Denmark), and tested using specified proportions $\mathrm{chi}^{2}$-tests, the sample did not differ significantly from the general public with regard to geographical region and gender. However, the sample underrepresented persons with a low level of education and low-income households. The sample also differed from census data with regard to age and obesity prevalence (self-reported $\mathrm{kg} / \mathrm{m}^{2}$ ). Compared to the latest national survey $(21)$ obese $(B M I \geq 30)$ and overweight persons (BMI 25-29.99) were slightly 
overrepresented. A BMI/age adjusted weight variable was computed according to reference data (21). After weighting, data were representative of the Danish population (aged 20-70) with regard to age, gender, region and BMI.

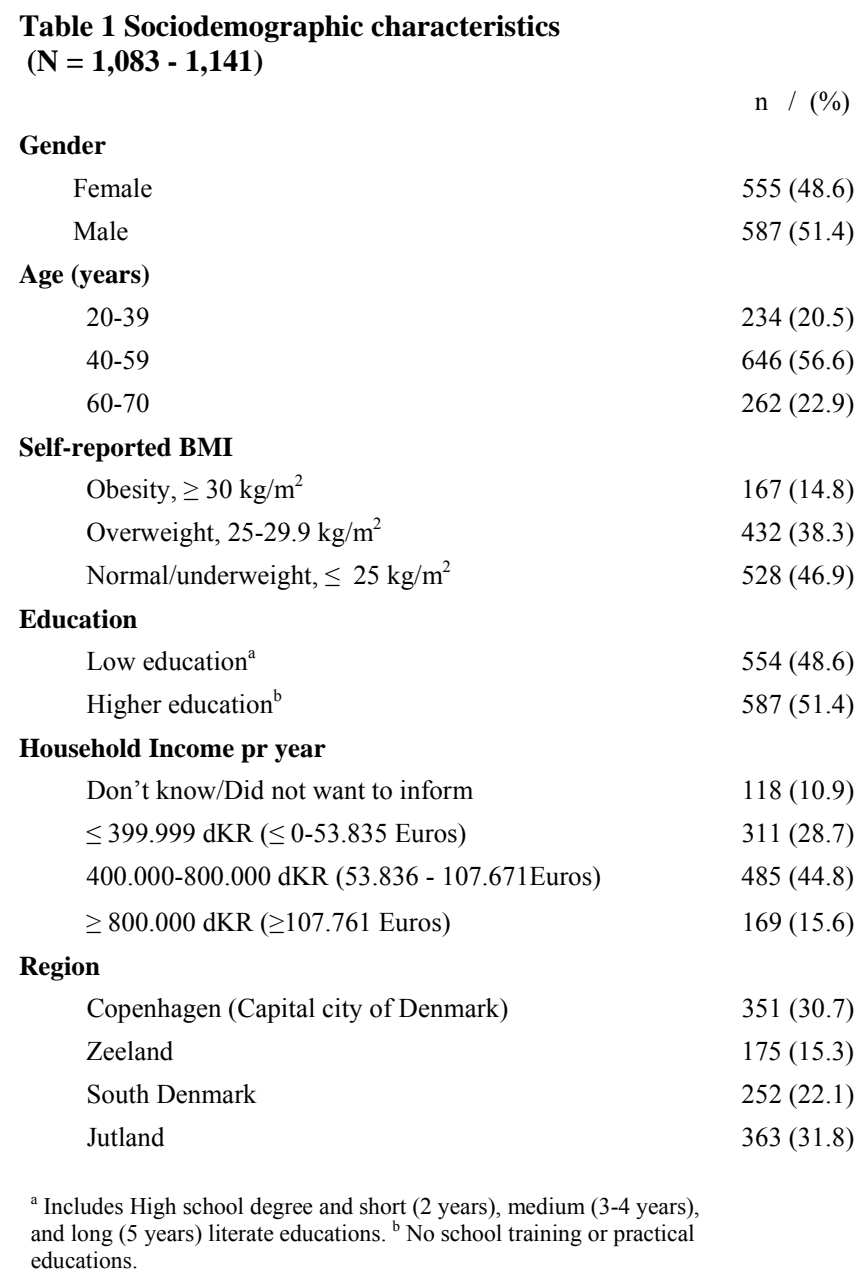

Attitudes to the public funding of obesity health care

This was assessed by asking participants for their views on the public funding of five obesity-related measures: weight-loss surgery, medical treatment, psychological therapy, dietary counselling, and informational campaigns. Three answer categories were offered to participants for each measure. These 
were: 'should be publicly funded', 'should be paid for by the individual', or 'don't know'. The actual wording was altered slightly where necessary. And in the case of informational campaigns the wording 'should be paid for by the individual' was replaced with 'the individuals should obtain the necessary information by themselves'. These response categories must be understood in a specifically Danish context, where most health care is provided within a public, state-administered universal system, which is financed almost exclusively by taxes. Although some health services depend fully or partly on individual payment, e.g. dental care, physiotherapy and medicinal expenses, in general no payments are required at the point of care, or earlier through e.g. insurances or co-payments for any form of hospital treatments, or consultations with physicians (22). Thus, prompting for further health care payment methods was irrelevant in this Danish study, whereas alterations or extensions of question wordings would be necessary in countries with different systems.

The five measures were selected so as to reveal attitudes to existing health care initiatives and also to test whether a distinction exists in the public mind between publicly funded prevention and similarly funded treatment of obesity. With the latter purpose in mind, two of the initiatives were carefully chosen to reflect rather pure instances of prevention and treatment, respectively: informational campaign was included as a preventative initiative, and weight-loss surgery was included as an example of treatment. The three remaining examples were expected to lie between these two examples, in the sense that they can be associated with both prevention and treatment.

It is possible additional reasons and arguments in favour of public funding of treatment may change some citizen's initial disapproving attitudes. In order to examine this, an extra question was put to the subgroup of respondents who had initially stated that the weight-loss surgery initiative should not be publicly funded, or who could not decide whether it should be. They were asked whether there were any conditions under which public funding would be acceptable. Respondents were allowed multiple responses with the following possible response categories: 'If the patient cannot afford to pay for the operation', 'If the obese condition is life threatening', 'If there is evidence that the patient is not responsible for the obesity', and 'If the obesity impairs the patient's social network and work capabilities'. 


\section{Obesity prejudice}

In order to assess the Dislike dimension of obesity prejudice, three scales from the Universal Measure of Bias (UMB) (16) were used: Negative Judgment, Distance, and Attraction. The 15 (3x5) items resulting from these subscales of the UMB were then employed. The UMB scale has shown adequate internal consistency and is strongly correlated with measures similar to the Dislike dimension. It was favoured over competing measures, since it covers three dimensions of bias / dislike and should therefore produce a more complete picture of Dislike. The fourth Equal Rights dimension of the UMB was not included - primarily in view of budget constraints.

In order to assess Perceived Control of body weight five attitudinal categories were used. Of these, four were taken from the Weight Control/Blame scale of the Antifat Attitude Test (15) ('There's no excuse for being fat'; 'If fat people really wanted to lose weight, they could'; 'The idea that genetics causes people to be fat is just an excuse'; 'Most fat people will latch onto almost any excuse for being fat'). These four items were selected in joint consideration of retaining items with the highest factor loadings from the original study and omitting items with wordings that were assessed as potentially transgressing this Perceived Control construct and approaching the Dislike constructs (such as 'If fat people knew how bad they looked, they would lose weight'). The fifth item ('Obesity is often selfinflicted') was devised by the authors.

The 15 items from UMB and the five items on Perceived Control were rated on the same 5-point Likert scale (Fully Disagree to Fully Agree). The original UMB study used a 7-point scale with slightly differently worded answers (16). Alterations were made to improve simplicity and intelligibility to the respondents.

All items replicated from earlier English studies were translated into Danish using a collaborative, iterative approach for translating questionnaires (23). This involved an initial translation of question 
items, a subsequent review from an independent researcher, followed by a final jointly conducted revision.

Initial reliability analyses revealed that the scales were acceptable indicators of Perceived Control (Cronbach's $\alpha=0.85)$ and Dislike, as the three Dislike subscales exhibited acceptable reliabilities ( $\alpha$ Negative Judgment $=0.92, \alpha$ Distance $=0.73, \alpha$ Attraction $=0.80$ ).

\section{Statistical analysis}

Univariate descriptive analysis was initially employed on the five obesity-related health care initiatives and on the follow-up question to the subgroup not supporting publicly funded weight-loss surgery.

In order to examine whether there was attitudinal distinction between preventive and treatment initiatives, a principal component analysis (using a varimax rotation) was employed on the five health care items. Items were coded with the values 1 for 'Public funding', 2 for 'don't know', and 3 for 'patient funding'. Based on the principal component analysis two variables measuring attitudes to publicly funded treatment and prevention, respectively, were extracted from computed factor scores for use in subsequent analysis; a higher score on these variables indicating less support.

To determine the influence of obesity prejudice on attitudes to public funding of obesity treatment and prevention, multiple mediation analysis was employed $(24,25)$. Following the assumptions of the attribution-value model, which links Perceived Control with Dislike, the three Dislike subscales were inserted as mediator variables and Perceived Control as a moderator variable. This procedure makes it possible to assess whether any of the Dislike subscales mediated the association between Perceived Control and attitudes to the public funding of obesity treatment and prevention, or whether alternatively, the Dislike subscales were significant when controlling for Perceived Control. Sobel's test for mediation (26) was used to assess the existence of a significant mediation effect. In any significant instances, the SPSS macro made available by Fairchild and colleagues (27) was applied in order to calculate effect sizes owing to mediating variables. 
In order to examine whether additional factors would adjust the effects of the prejudicial factors and further explain the variation of the dependent variable, follow-up regression analyses were conducted. The three Dislike subscales and the Perceived Control scale were included as predictors together with age, self-reported BMI $\left(\mathrm{kg} / \mathrm{m}^{2}\right)$, gender, education, and household income. In addition, three further attitude items were included: one of which was political orientation (Left, Right, or Other). This was a re-coding of the respondent's intention to vote for either a Danish political party on the left side of the political scale, or a party on the right side, or other decision ('Don't know', 'Not planning to vote' and so on). Two further items on attitudes to the scope of public health care duties were included. The first was intended to tap into general attitudes to universal health care: 'The public health system has an obligation in principle to treat all kinds of disease' and the second item was to reveal attitudes to public duties when diseases are self-inflicted: 'The public purse should not fund the treatment of diseases when patients are responsible for the condition'. Both items were 5-point Likert scales (ranging from 1. 'fully disagree' to 5. 'fully agree') and were treated as continuous variables in the analysis. Categorical variables (gender, education, household income, and political orientation) were dummy coded. A backwards stepwise regression modelling approach was employed, which removed the least significant variables one by one until all variables in the equation were significant at the 0.05 significance level.

\section{Results}

Public opinion about collectively funded obesity health care

Descriptive results from the five items pertaining to the public funding of obesity treatments showed that weight-loss surgery and medical treatment were more or less equally rated. Only $33.3 \%$ were of the opinion that the former should be funded by the public, $46.5 \%$ thought that the patient should pay, and $20.3 \%$ responded 'Don't know'. For medical treatment there were rather similar percentages - see Table 2(a). By contrast, the three remaining examples were supported by a majority of respondents, the figures being: psychological therapy (51.6\%), dietary counselling (67.8\%), and informational campaigns $(83.8 \%)$. 
Public attitudes to obesity health care

Table 2 Descriptive statistics ${ }^{\mathrm{a}}$

(a) Attitudes to public funding of obesity-related health care initiatives ${ }^{b}$ $(\mathrm{N}=1,127)$

Weight-loss surgery

Public funding

Individual funding

Don't know

Medical treatment

Public funding

Individual funding

Don't know

Psychological therapy

Public funding

Individual funding

Don't know

Dietary counselling

Public funding

Individual funding

Don't know

Informational campaigns

Public funding

Individual funding

Don't know

(b) Exceptions to public funding of weight-loss surgeries among subgroup (66.8\%) not supporting public funding initially ${ }^{c}$

If the patient cannot afford to pay for the operation

If the obese condition is life threatening

If there is evidence that the patient is not responsible for the obesity

If the obesity impairs the patient's social network and work capabilities
Institute of Food and Resource Economics

University of Copenhagen

This is a post-print version of an article published in Obesity - the official journal of The Obesity Society

${ }^{a}$ Number of respondents reduced to $\mathrm{N}=1,127$ because it was not possible to compute weighted values in 14 cases, due to unreliable BMI values. ${ }^{\text {b }}$ Introductory text to all five questions: 'Which of these statements do you agree most with?'. Answer wordings followed this form: 'The public should pay for the weight-loss surgery.', 'It should be the patient who pays for the weight-loss surgery.', and 'Don't know'. 'A multiple response question with the introductory text: 'The public should only pay for weight-loss surgery' followed by the answering categories displayed in the table.

When the subgroup of respondents who did not initially support publicly funded weight-loss surgery, were asked whether there were any conditions under which public funding would be acceptable, $74.5 \%$ changed their mind - with the proviso that evidence can be provided that the obesity was not caused by the individual's own conduct - see Table 2(b). 47.2\% took the fact that a condition is life-threatening to 
be important enough to shift their stance to one of support for publicly funded weight-loss surgery. Thus, a life-saving argument for obesity treatment changed the acceptability rate substantially, with the level of support for publicly funded weight-loss surgery rising from an initial 33\% to approximately $65 \%$.

\section{Do attitudes to treatment and prevention differ?}

Results of the principal component analysis gave two components with eigenvalues above 1.0 (see table 3). Further inspection showed that the first component expressed a treatment factor, since surgery and medical treatment of obesity had very high factor loadings whereas informational campaign did not. The second component expressed a preventive factor, as opposite loading characteristics were found. At the same time dietary and psychological initiatives loaded relatively highly on both factors. Thus, the public appears to interpret them as having both preventive and treatment-like properties.

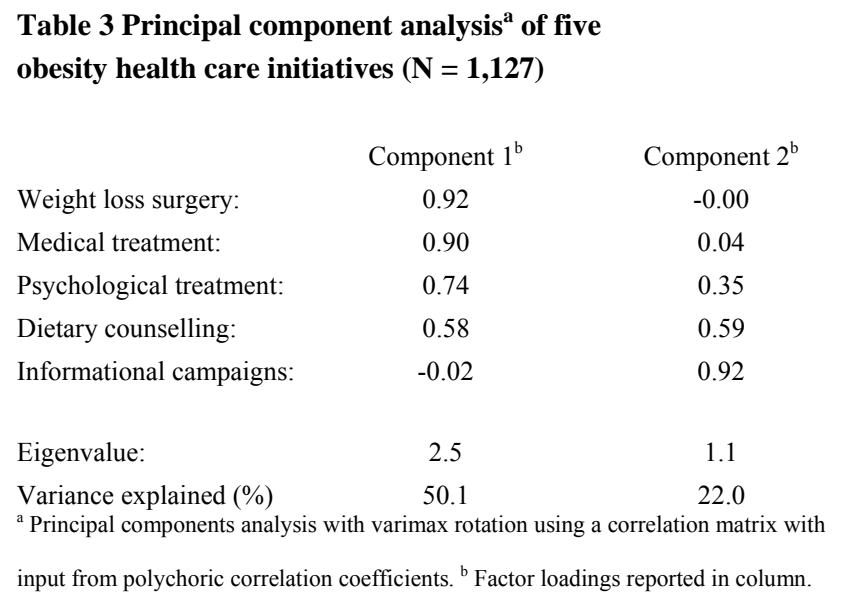

Dislike and Perceived Control in attitudes to public spending on obesity treatment

Bivariate analyses showed that Negative Judgment $(\mathrm{r}=0.23, \mathrm{P}<0.001)$, Distance $(\mathrm{r}=0.22, \mathrm{P}<0.001)$, and (non)Attraction $(\mathrm{r}=0.29, \mathrm{P}<0,001)$ were positively correlated with disapproval of public funding for obesity treatments. Perceived Control $(\mathrm{r}=0.52, \mathrm{P}<0.001)$ also had a positive association with 
disapproval of public funding for obesity treatment. Multiple mediation analysis revealed that neither Negative Judgment $(\beta=0.002, \mathrm{P}=0.96)$ nor Distance $(\beta=0.012, \mathrm{P}=0.70)$ had a significant effect on attitudes to the public funding of obesity treatment when we controlled for Perceived Control. (Non)Attraction did, however, have a significant effect $(\beta=0.075, \mathrm{P}=0.012)$ and Sobel's test for mediation showed that it significantly mediated the effect of Perceived Control $(Z=2.83, P=0.005)$. However, this was only a partially mediated effect. Thus, of the approximate $28 \%$ of the variation that (non)Attraction and Perceived Control explained together, over 20\% was from the direct effect of Perceived Control, whilst around 8\% was explained by (non)Attraction and Perceived Control together, and less than $0.1 \%$ was explained solely by (non)Attraction.

Turning to the follow-up regression analysis, the predictors in the final model explained approximately $35 \%$ (adjusted $\mathrm{R}^{2}=0.351$ ) of the variance in attitudes to the public funding of obesity treatment. Perceived Control was the strongest predictor $(\beta=0.399, \mathrm{P}=0.001)$, followed by the attitude statement 'The public purse should not fund the treatment of diseases when people are responsible for the condition' $(\beta=0.272, \mathrm{P}=0.001)$. Self-reported BMI $(\beta=-0.102, \mathrm{P}=0.001)$ and agreeing that 'The public purse has an obligation in principle to treat all kinds of disease' $(\beta=-0.066, \mathrm{P}=0.006)$ also predicted attitudes significantly, and as would be expected the relationships were negative in these two instances. None of the Dislike subscales turned out to be a significant predictor when the other factors in the final regression model were controlled for.

Dislike and Perceived Control in attitudes to public spending on obesity prevention

Initial bivariate analyses showed that Perceived Control $(\mathrm{r}=0.05, \mathrm{P}=0.08)$ did not attain statistical significance. Given this non-significant effect, mediation analysis becomes superfluous (24) and it was therefore abandoned.

In the follow-up regression analysis, only a relatively small part of the variance in the dependent variable was explained by the predictor variables (adjusted $\mathrm{R}^{2}=0.031$ ). Significant predictors were the Negative Judgment subscale $(\beta=0.83, \mathrm{P}=0.008)$, age $(\beta=0.88, \mathrm{P}=0.004)$ and voting for a Right wing 
party, compared to Left wing voting $(\beta=0.116 \mathrm{P}=0.001)$. Household income also had a significant effect, since high income households were less likely to disapprove of public funding, than households in which income was not reported $(\beta=0.105, \mathrm{P}=0.006)$. Perceived Control retained its non-significant effect.

\section{Discussion}

The purpose of this study was to assess support for publicly funded obesity treatment and prevention among the Danish public, and to investigate whether Dislike and/or Perceived Control predicted attitudes in this arena. The results confirm findings set out in other population-based surveys, which show relatively high levels of public support for prevention initiatives $(3,5,7)$. It was also shown that the public distinguishes between the treatment and prevention of obesity, and additionally that there are considerable differences in the approval rates thereof. Thus, pure obesity treatment initiatives were supported much less strongly than preventive measures. It should be noted, however, that a sizeable proportion of the participants who initially disapproved of public funding for weight-loss surgery changed their minds when told that it was being conducted for life saving reasons.

The disclosure of a non-significant association between Perceived Control and support for preventative measures in this study is largely in line with two earlier studies in which only small effects were found $(5,6)$. Furthermore, Dislike had no significant effect on the attitude to public funding of treatment initiatives and only a small direct effect on the attitude to funding of prevention. It was a new finding that Perceived Control predicts attitudes to obesity treatment, with a large effect size $(r=0.52)$. Since agreeing that '...the public should not fund health care when people are personally responsible for their condition' was an additional powerful predictor, this study elucidates the overall importance of responsibility amongst the public, when it comes to obesity treatment.

It should be noted that the latter is contrary to a recent finding in which only Dislike had a significant effect on denying surgery to overweight patients in Canada (18). However, this study was based on a student sample, which may not be representative of the general public in its obesity prejudices. More 
studies should be conducted to explore whether these conflicting results are related to the population surveyed, or to questionnaire specific aspects.

A limitation of this study is that respondents were not posed questions that could have clarified their understanding of the complex etiology of obesity (28). Since the beliefs people hold about the causes of obesity, such as heredity and environmental factors, have been shown to determine attitudes to obesity prevention in earlier studies $(3,6)$, any interplay with Perceived Control remains unobserved. Not all members of the general Danish public, in particular the elderly above 70 years, use the internet. Consequently, since this survey is based on a web-questionnaire, it is only possible to claim representativeness for Danish citizens between 20 and 70 years of age. Additionally, the sample also underrepresented low educated persons and low-income households. Although these factors had only a slight, or no effect, on the dependent variables, support rates could have differed slightly. The response rate of $30.9 \%$ is a further limitation of this study, since low response rates may create non-response error in means and proportions, although studies have shown that the lowered response rates in recent years only slightly affect demographic representativeness (29) and differences in proportions (30). Finally, the omission of subscales and items from previously developed instruments may potentially affect the validity of these. Roughly speaking, similar correlation coefficients were found between Perceived Control and the three Dislike subscales (Negative Judgment $r=0.42, \mathrm{P}<0.001$; Distance $\mathrm{r}=0.35, \mathrm{P}<0.001$; Attraction $\mathrm{r}=0.43, \mathrm{P}<0.001)$, as in earlier studies of these constructs $(12-15,17,18)$. This suggests that scale validity was not seriously impaired.

Notwithstanding these reservations, the effect of the responsibility factors on attitudes to publicly funded obesity treatment was so strong in the study reported here that it is unlikely to be coincidental. This finding is noteworthy, considering the almost all-inclusive consensus in the scientific community that the causes of obesity are multi-factorial and certainly not only attributable to deliberate individual choices (28). This may in turn have the consequence that future health care decisions pertaining to obesity are made in response to pressure from a public with a limited understanding, or even misconception, of the diverse causes of obesity. 
Based on the wish that public debates regarding obesity health care should rest on sound information, it can be argued that information focusing on the external causes of obesity, should be disseminated to a wider public in order to elevate the public's understanding of obesity in general (11) and health care initiatives in particular $(6,9)$.

But no matter how important public information may be in straightening out misconceptions, western countries rest on a historically robust cultural belief in self-determination. Citizens with this conviction are in general less inclined to back up social welfare and equality measures and more inclined to stress free enterprise and social Darwinism (31). And indeed, it has been shown that the propensity to attribute obesity to lack of willpower correlates strongly with classic Protestant values (14).

According to the data in this study, a rather large group of Danish citizens attributes obesity to personal responsibility. In all, 55\% agreed that 'If fat people really wanted to lose weight, they could', whilst $30.2 \%$ agreed with the categorical statement: 'There is no excuse for being fat'. The prevalence of views like these, together with real life experiences and strong convictions about the importance and potential of self-reliance and self-control, suggests that reversing the belief that obesity is self-inflicted may be a very difficult task.

This circumstance suggests that a wider ethical discussion regarding the relevance of collectively funded obesity health care is called for. Such a discussion should take the performance of different financing systems under examination, while considering the overall goals of health care (1). We will not go into this complex discussion here, but rather focus on the challenges this constitutes for contemporary decision makers. Our study suggests that politicians in countries with a universal health care system, like Denmark, are at odds with the public mind. If decision makers wish to ensure equal access to all forms of health care they may look for arguments to the effect that collective health care should also cover diseases, such as obesity, that are thought to be self-inflicted. One path would be to argue that if untreated, obesity will lead to further conditions, covered by the system, which will be much more costly to treat. Also, a more combative ethical stance may be taken by defending the 
classical humanitarian view that everyone is entitled to health care irrespective of the circumstances. A different path would involve recognizing the concerns that many citizens have by incorporating responsibility aspects into the organization and funding of collective health care. The appropriate way of doing this would differ from country to country. In countries with state-administered universal health care, a mechanism in which taxes are put on high-risk food stuffs of which obese people are likely to eat more (28) seems to represent a viable way forward (32).

The research reported here shows that collective funding of known and applied treatments methods for severe obesity is disapproved of by a majority of the Danish public. The disapproval can be primarily explained by responsibility factors, such as perceived control of body weight. The low importance of Dislike may possibly be explained by the circumstance that the questionnaire employed self-reported evaluations, thus revealing so-called explicit, as opposed to implicit, attitudes (33). Since outspoken prejudice is not socially acceptable today, prejudicial segments could have avoided answering the Dislike items truthfully by ticking off non-prejudicial response categories. Others may not have been unaware of their prejudice. If this is the case, the real effect from Dislike is underestimated in this study. Future research, which examines public attitudes to obesity health care, would contribute to answering this unresolved question by incorporating implicit measures of antifat attitudes (12).

\section{Acknowledgements}

We thank Ola Ekholm for providing reference data necessary for applying a BMI/age adjusted weight variable; Niels Toft for helpful comments on the statistical analyses employed; and Paul Robinson and Stuart Wright for language editing. The study reported is financed by the Danish Agency for Science, Technology and Innovation under the Ministry of Science, Technology and Innovation.

\section{Reference List}

1. WHO. The World Health Report 2000. Health Systems: Improving Performance. Geneva, World Health Organization, 2000, pp 1-215. 
2. Barry CL, Brescoll VL, Brownell KD, Schlesinger M. Obesity Metaphors: How Beliefs about the Causes of Obesity Affect Support for Public Policy. Milbank Q. 2009;87:7-47.

3. Hilbert A, Rief W, Braehler E. What determines public support of obesity prevention? $J$ Epidemiol Community Health. 2007;61:585-90.

4. Kaiser et al. Survey on Childhood Obesity. San Jose Mercury News, 2004.

5. Oliver JE, Lee T. Public opinion and the politics of obesity in America. J Health Polit Policy Law. 2005;30:923-54.

6. Hilbert A, Rief W, Braehler E. Stigmatizing attitudes toward obesity in a representative population-based sample. Obesity (Silver Spring). 2008;16:1529-34.

7. Fuemmeler BF, Baffi C, Masse LC, Atienza AA, Evans WD. Employer and healthcare policy interventions aimed at adult obesity. Am J Prev Med. 2007;32:44-51.

8. Hardus PM, van Vuuren CL, Crawford D, Worsley A. Public perceptions of the causes and prevention of obesity among primary school children. Int J Obes Relat Metab Disord. 2003;27:1465-71.

9. Evans WD, Renaud JM, Finkelstein E, Kamerow DB, Brown DS. Changing perceptions of the childhood obesity epidemic. Am J Health Behav. 2006;30:167-76.

10. National Institutes of Health/National HeartLung and Blood Institute, North American Association for the Study of Obesity. Practical Guide to the Identification, Evaluation, and Treatment of Overweight and Obesity in Adults. Bethesda, Md., National Institutes of Health, 2000., pp 1-40

11. Puhl RM, Heuer CA. The Stigma of Obesity: A Review and Update. Obesity (Silver Spring). 2009;17:941-64. 
12. O'Brien KS, Latner JD, Halberstadt J, Hunter JA, Anderson J, Caputi P. Do Antifat Attitudes Predict Antifat Behaviors? Obesity (Silver Spring). 2008;16:S87-S92.

13. Allison DB, Basile VC, Yuker HE. The Measurement of Attitudes Toward and Beliefs About Obese Persons. Int J Eat Disord. 1991;10:599-607.

14. Crandall CS. Prejudice Against Fat People - Ideology and Self-Interest. J Pers Soc Psychol. 1994;66:882-94.

15. Lewis RJ, Cash TF, Jacobi L, BubbLewis C. Prejudice toward fat people: The development and validation of the antifat attitudes test. Obesity (Silver Spring). 1997;5:297-307.

16. Latner JD, O'Brien KS, Durso LE, Brinkman LA, MacDonald T. Weighing obesity stigma: the relative strength of different forms of bias. Int J Obes (London). 2008;32:1145-52.

17. Vartanian L. Disgust and perceived control in attitudes toward obese people. Int J Obes (London). 2010; E-pub Ahead of print 2 March 2010; doi:10.1038/ijo.2010.45.

18. Brochu PM, Esses VM. Weight Prejudice and Medical Policy: Support for an Ambiguously Discriminatory Policy Is Influenced by Prejudice-Colored Glasses. Anal Soc Iss Pub Pol. 2009;9:117-33.

19. Crandall CS, D'Anello S, Sakalli N, Lazarus E, Wieczorkowska G, Feather NT. An attributionvalue model of prejudice: Anti-fat attitudes in six nations. Pers Soc Psych Bull. 2001;27:30-7.

20. Guidelines from The Danish National Comitee on Biomedical Research Ethics. http://www.cvk.sum.dk/English/guidelinesaboutnotification.aspx\#AFSNIT\%202.0. Retained November $10^{\text {th }} 2010$; Latest update: April $16^{\text {th }} 2009$

21. Ekholm O, Hesse U, Davidsen M, Kjoller M. The study design and characteristics of the Danish national health interview surveys. Scand J Public Health. 2009;37:758-65. 
22. Vallgårda S, Krasnik A, Vrangbæk K. Health Care Systems in Transition. Denmark. European Observatory on Health Care Systems WHO: Copenhagen, 2001, pp 1-92.

23. Douglas SP, Craig CS. Collaborative and iterative translation: An alternative approach to back translation. J Int Mar. 2007;15:30-43.

24. Baron RM, Kenny DA. The Moderator Mediator Variable Distinction in Social PsychologicalResearch - Conceptual, Strategic, and Statistical Considerations. J Pers Soc Psychol. 1986;51:1173-82.

25. MacKinnon, DP. Multiple Mediator Model. In: Introduction to Statistical Mediation Analysis. 1st edn. New York, NY, Lawrence Erlbaum Associates, 2008, pp 103-126.

26. Sobel ME.Asymptotic confidence intervals for indirect effects in structural equation models. In S. Leinhardt (Ed.), Sociological Methodology 1982 (pp. 290-312). Washington DC: American Sociological Association.

27. Fairchild AJ, MacKinnon DP, Taborga MP, Taylor AB. R-2 effect-size measures for mediation analysis. Behav Res Methods. 2009;41:486-98.

28. World Health Organization. Part III. Understanding how overweight and obesity develop. In: WHO Technical Report Series 894. Obesity. Preventing and managing the global epidemic. WHO: Geneve, 2000, pp 100-152.

29. Holbrook A, Krosnick J, Alison P. The Causes and Consequences of Response Rates in Surveys by the News Media and Government Contractor Survey Research Firms. In: Lepkowski JM, Tucker NC, Brick JM, et al (eds). Advances in telephone survey methodology, Wiley: New York, 2007, pp 499-528.

30. Keeter S, Kennedy C, Dimock M, Best J, Craighill P. Gauging the Impact of Growing Nonresponse on Estimates from a National RDD Telephone Survey. Public Opin Q. 2006;70:759-779. 
31. Frey RA, Powell LA. Protestant Work Ethic Endorsement and Social Justice Values in Developing and Developed Societies Comparing Jamaica and New Zealand. Psych Dev Soc. 2009;21:51-77.

32. Cappelen AW, Norheim OF. Responsibility in health care: a liberal egalitarian approach. $J$ Med Ethics. 2005;31:476-80.

33. Greenwald AG, McGhee DE, Schwartz JLK. Measuring individual differences in implicit cognition: The implicit association test. J Per and Soc Psychol. 1998; 74;1464-1480. 